\title{
Nobelist TD LEE Scientist Cooperation Network and Scientist Innovation Ability Model
}

\author{
Jin-Qing Fang and Qiang Liu \\ China Institute of Atomic Energy, Beijing 102413, China \\ Correspondence should be addressed to Jin-Qing Fang; fjq96@126.com
}

Received 24 July 2013; Accepted 7 August 2013

Academic Editor: Guanghui Wen

Copyright (C) 2013 J.-Q. Fang and Q. Liu. This is an open access article distributed under the Creative Commons Attribution License, which permits unrestricted use, distribution, and reproduction in any medium, provided the original work is properly cited.

\begin{abstract}
Nobelist TD Lee scientist cooperation network (TDLSCN) and their innovation ability are studied. It is found that the TDLSCN not only has the common topological properties both of scale-free and small-world for a general scientist cooperation networks, but also appears the creation multiple-peak phenomenon for number of published paper with year evolution, which become Nobelist TD Lee's significant mark distinguished from other scientists. This new phenomenon has not been revealed in the scientist cooperation networks before. To demonstrate and explain this new finding, we propose a theoretical model for a nature scientist and his/her team innovation ability. The theoretical results are consistent with the empirical studies very well. This research demonstrates that the model has a certain universality and can be extended to estimate innovation ability for any nature scientist and his/her team. It is a better method for evaluating scientist innovation ability and his/her team for the academic profession and is of application potential.
\end{abstract}

As is well known, scientist cooperation networks as an important issue in network science have been studied widely [18]. It is significant to study Nobelist Tsung-Dao Lee (TD Lee, Chinese: 李政道) scientist cooperation networks, which is a typical nature scientist team. TD Lee is a celebrated Chineseborn American physicist, well known for his work [9] on parity violation, the Lee Model, particle physics, relativistic heavy ion (RHIC) physics, nontopological solitons, and soliton stars. In 1957, TD Lee, at the age of 30, won the Nobel Prize in Physics with CN Yang (Chinese: 杨振宁) for their work on the violation of parity law in weak interaction, which Chien-Shiung Wu experimentally verified.

According to Lee Digital Library [9], we have constructed and studied TD Lee scientist cooperation networks (TDLSCN), as shown in Figure 1, where the nodes are TD Lee and his cooperators, and two nodes are connected if two scientists have written an article together. The TDLSCN includes published 195 papers and 92 network nodes during 1948-1996. TD Lee (李政道) and CN Yang (杨振宁) are two hubs in the TDLSCN, in which 37 papers were cooperated with CN Yang, both of them awarded the Nobel Prize in Physics at 1957. Table 1 and Figures 2 and 3 show the common topological properties and main features for the TDLSCN, respectively.

It can be seen from Figures 1 and 2 and Table 1 that the TDLSCN has general topological properties, that is, scale-free property with 3 power laws: the degree exponent $\gamma=2.5436$, the node strength exponent $\gamma_{s}=2.3613$, and the edge weight exponent $\gamma_{w}=2.1786$, as well as small-world effect: the shortest path length $L=2.588758$ and clustering coefficient $C=0.406680$.

It is found from Figure 3 that the most important feature of the TDLSCN is the multiple peak phenomenon for number of published papers with year evolution, or so-called creation multiple-peak for a nature scientist lifetime, which is revealed for the first time and is not explained yet at home and abroad. One model for the creativity of scientific knowledge was proposed by Soler [10], but he has not found and cannot explain the creation multiple peak at all. In addition, Wen and his cooperators proposed a weighted local-world evolving network model with aging nodes [11] and revealed scale-free as well as small-world properties in the model but did not have a chance to find creation multiple-peak phenomenon yet. 


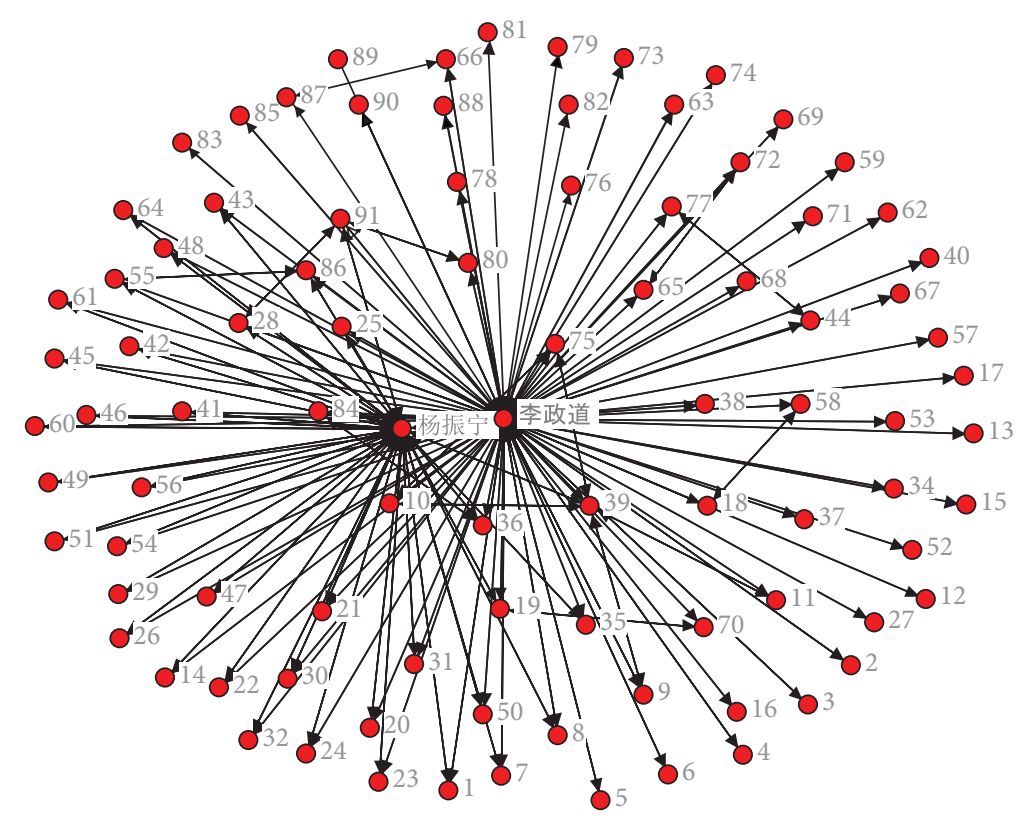

FIGURE 1: TD Lee scientist cooperation network.

TABLE 1: Main topological properties of the TDLSCN.

\begin{tabular}{lcccc}
\hline $\begin{array}{l}\text { Degree } \\
\text { exponent } \gamma \\
\text { exponent } \gamma_{s}\end{array}$ & $\begin{array}{c}\text { Node } \\
\text { strength }\end{array}$ & $\begin{array}{c}\text { Edge-weighted } \\
\text { exponent } \gamma_{w}\end{array}$ & $\begin{array}{c}\text { Shortest } \\
\text { path length } \\
L\end{array}$ & $\begin{array}{c}\text { Clustering } \\
\text { coefficient } C\end{array}$ \\
\hline 2.5436 & 2.3613 & 2.1786 & 2.588758 & 0.406680 \\
\hline
\end{tabular}

To explain the creation multiple peak phenomenon above, we propose a theoretical model of innovation ability for a nature scientist and his/her team. Our model is based on the network of citations among research articles and mainly includes two parts of contribution, one is the first author (TD Lee) contribution in his papers and the second is the first author contribution contained in the cooperation papers, and it considered their citations and influence factors of the journal published papers in the TDLSCN. The model allows assigning to each article a positive value for its creativity, that is, for its creation of new knowledge. Therefore, the innovation ability for a nature scientist and his/her team should obey following dynamical equations, respectively:

$$
\begin{gathered}
c_{i}=\sum_{m}^{n m_{i}} w(i, m) \alpha_{i, m} r_{i, m}+\sum_{k}^{n k_{i}}(1-g(i, k)) \beta_{i, k} r_{i, k}, \\
C_{i, \text { team }}=\sum_{m}^{n m_{i}} \alpha_{i, m} r_{i, m}+\sum_{k}^{n k_{i}} \beta_{i, k} r_{i, k},
\end{gathered}
$$

where $w(i, m)$ and $g(i, k)$, respectively, are the weighted probability with $m$ number of papers in the $i$ year for the first author and the other contributions for $k$ number of cooperator papers. We consider that the first author TD Lee's weighted probability should be bigger than his cooperators, which is reasonable and may be taken as follows: $0.5 \leq$ $w(i, m) \leq 1,0.2 \leq g(i, k)<0.8$ by random selection; $n m_{i}, n k_{i}$ are the number of papers selected by SCI published journal for the first author and cooperators, respectively. $\alpha_{i, m}$ and $\beta_{i, k}$ are influence factors of the journal published papers for the first author and cooperators. $r_{i, m}$ and $r_{i, k}$ are SCI times cited, respectively, for the first author TD Lee and his cooperators.

It is noted that there is a hypothesis in our model that any reference's contribution only is a very small part for the whole paper, so it can be canceled. According to (1)(2), we have carried out 1000 random calculations, and then average innovation ability of TD Lee, collaborators, and his team can be obtained, respectively. Figures 4, 5, and 6 show the theoretical results with multiple peak for the innovation ability of TD Lee and his team, which are consistent with the empirical studies very well.

The cumulative creative ability $C_{\text {year }}$ of a scientist and $C_{\text {total }}$ of his/her team in merit of research are expressed respectively as follows:

$$
\begin{gathered}
C_{\text {year }}=\sum_{i=1}^{p} C_{i} \quad \text { For the first scientist, } \\
C_{\text {total }}=\sum_{i=1}^{p} C_{i, \text { team }} \quad \text { For the scientist team, }
\end{gathered}
$$

where $i=1$ is a normalized beginning year of published paper and $p$ is a number of ending years of publication, for example, TD Lee has $p=48$ since his beginning year of published paper is 1948 and ending year is 1996.

The cumulative and single creative abilities for an author and his/her team are proposed as an indicator of his or her merit of research. Figure 7 shows the comparison of cumulative creative ability with single-year creative ability for TD Lee (a) and his team (b), respectively.

It can be seen from Figures 4-7 that main new features of TDLSCN are as follows. 


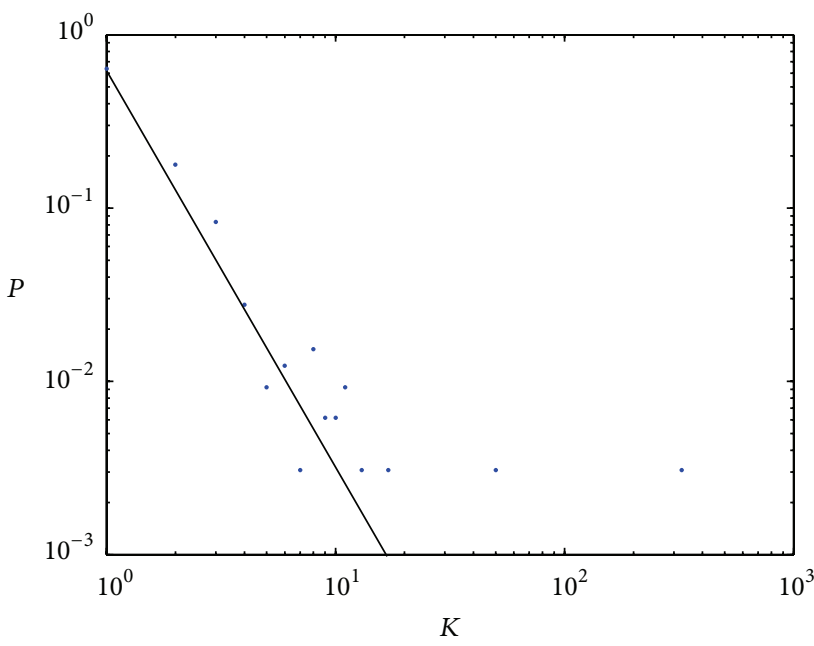

(a)

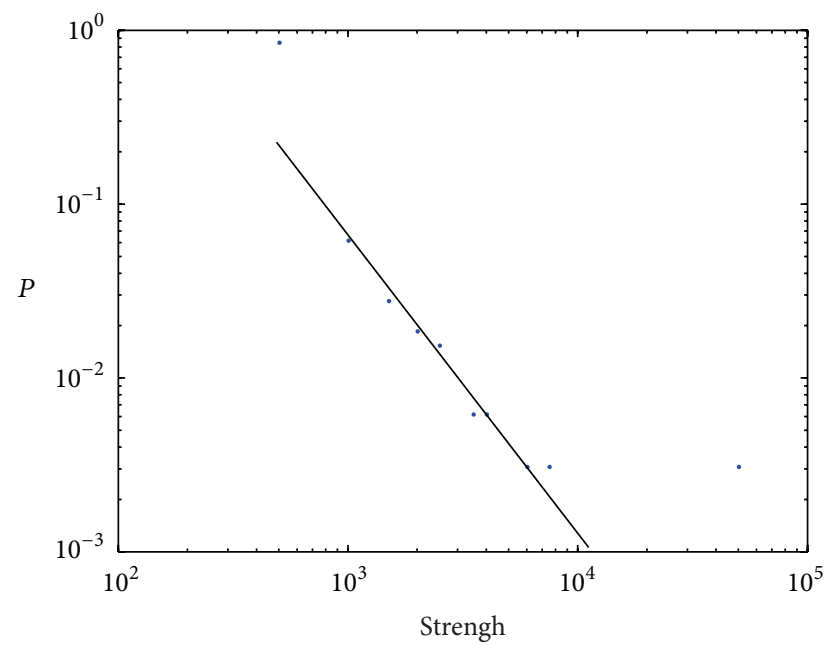

(b)

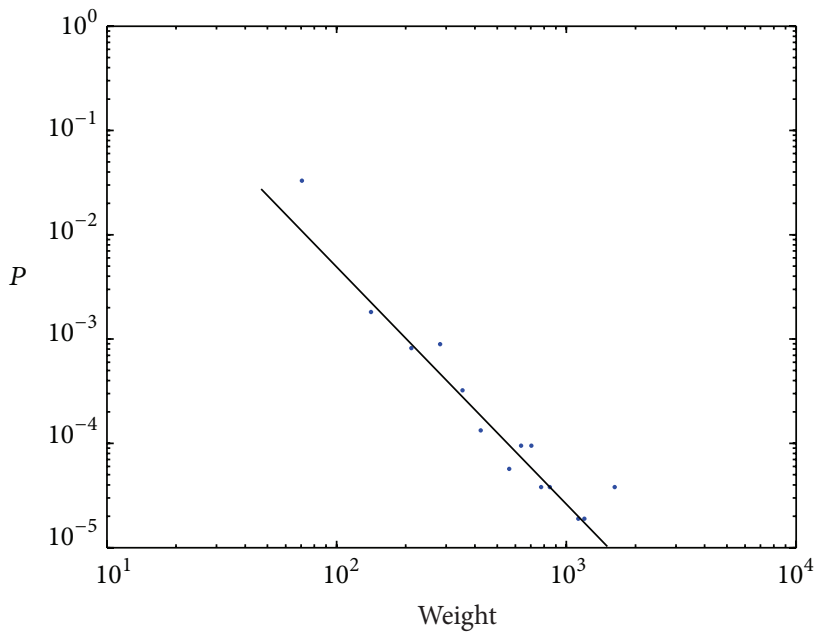

(c)

FIGURE 2: Topological property for TD Lee scientist cooperation network. (a) Node degree distribution; (b) node strength distribution; (c) edge weight distribution.

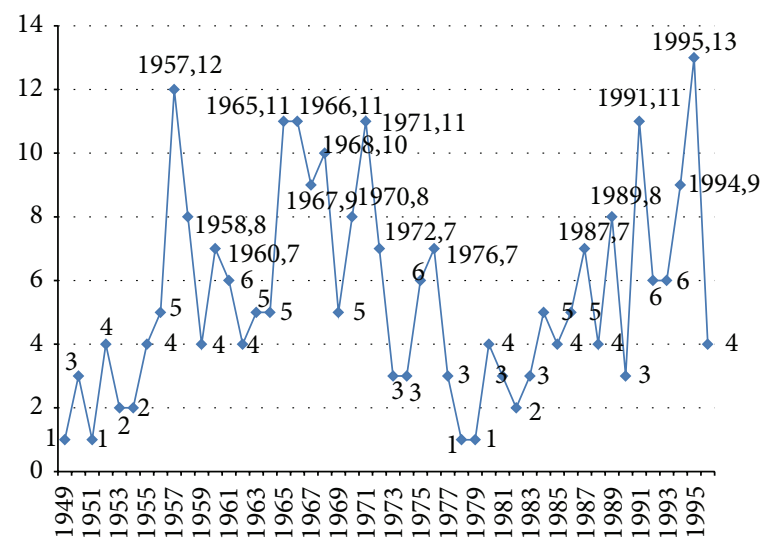

FIGURE 3: Total cooperative article counts $N$ versus year evolution. 


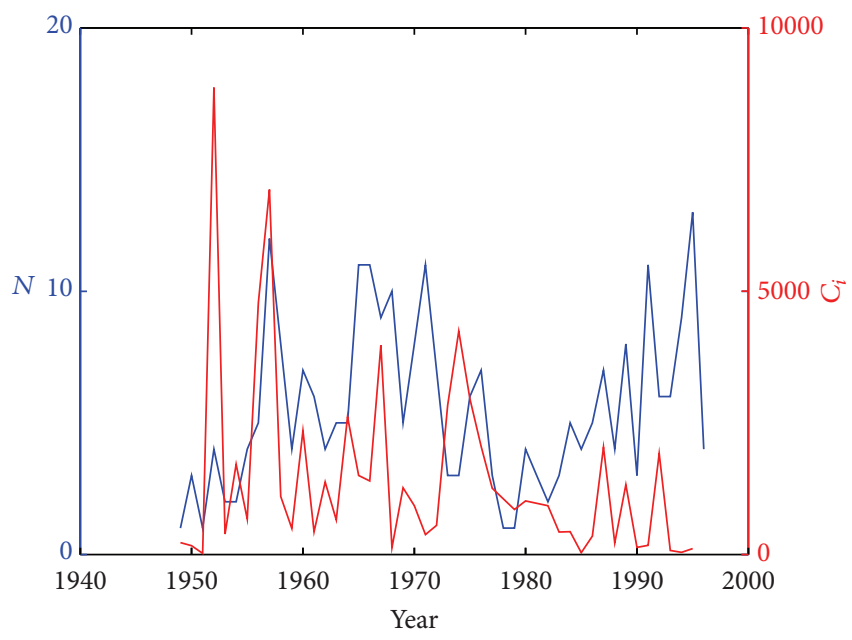

(a)

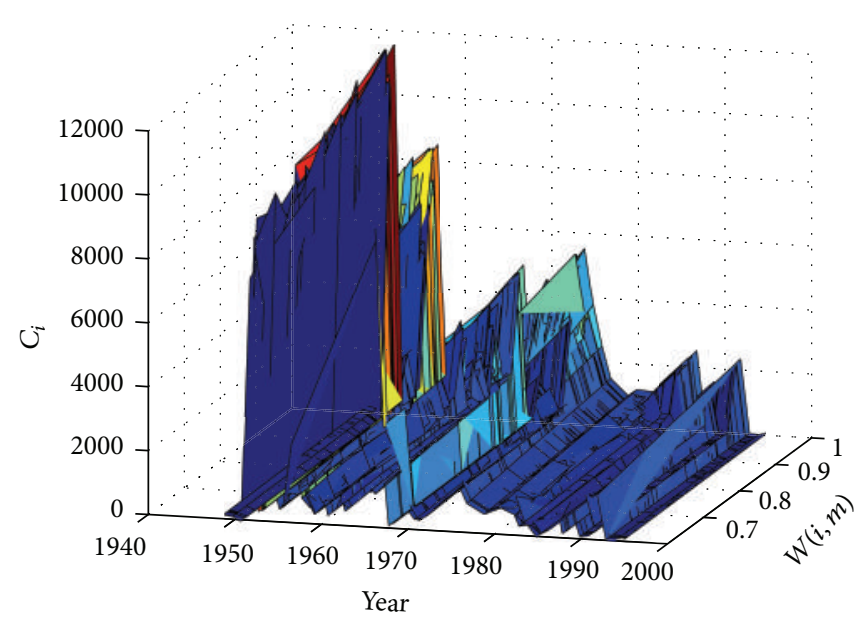

(b)

FIgure 4: Total articles $N$ and innovation ability $C_{i}$ for TD Lee. (a) $N$ and $C_{i}$ versus year; blue line denotes total number of papers; red line is creative ability; (b) $\left(C_{i}, W(i, m)\right)$ versus year.

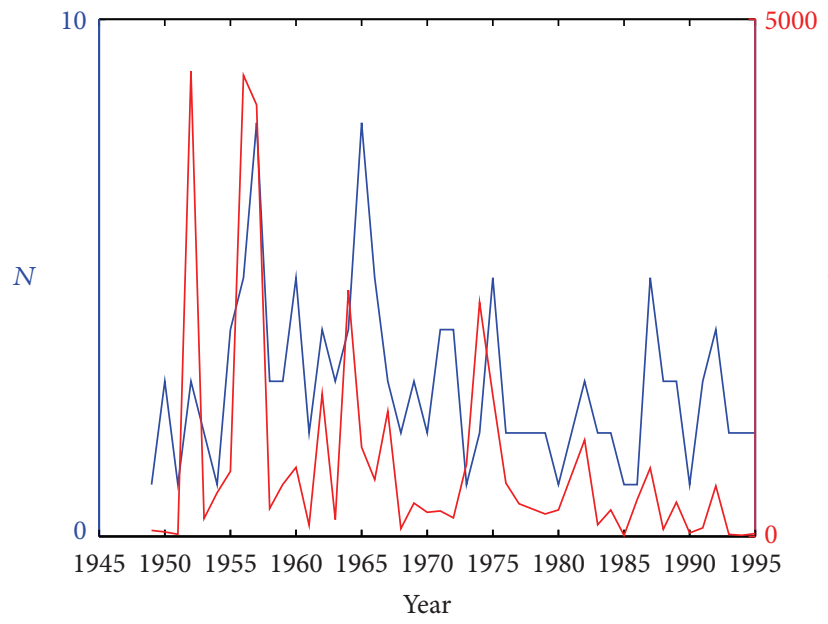

(a)

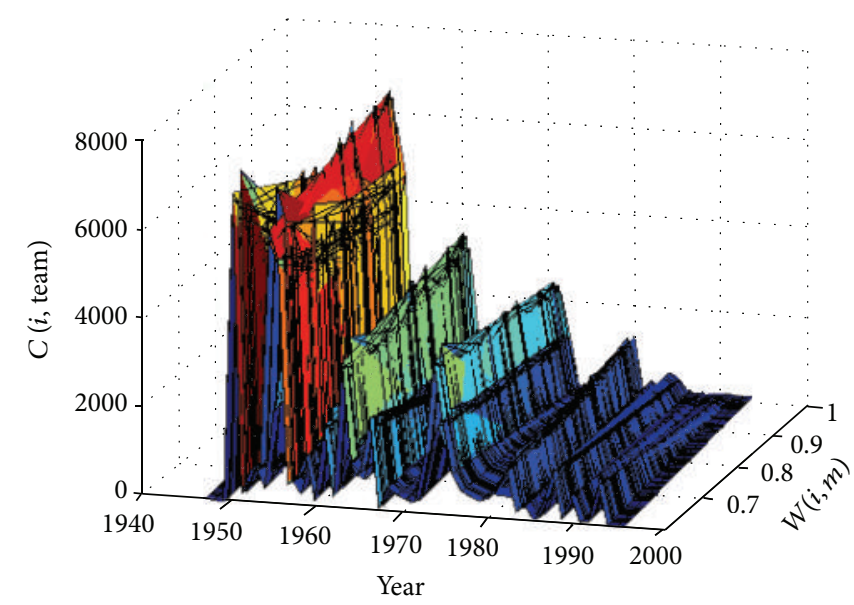

(b)

Figure 5: Total articles $N$ and innovation ability $C_{i}$ for TD Lee's cooperator. (a) $N$ and $C_{i}$ versus year; (b) $(C(i$, team), $W(i, m))$ versus year.

(1) Creation multiple peak phenomenon for number of published articles versus year for a nature scientist is found for the first time. TD Lee and his cooperation team are only taken as the most typical example for explanation. This demonstrates that this theory is found to be in line with the empirical statistical results. Both of them have one-for-one corresponding relations. The maximum number of multiple peaks for published papers is 12 in 1957 and 13 in 1995 for TD Lee.

(2) The results of theoretical calculations are as follows. The highest peak value of creative ability $C=9000$ for TD Lee, 4500 for collaborators, and 13500 for his team, respectively. Total cumulative innovation ability is $C=66347$ for the TD Lee, $C_{\text {other }}=32659$ for his collaborators, and $C_{\text {team }}=99006$ for his cooperation team, respectively. In fact, there are nonlinear complex relations with multiple factors for innovation ability; that is, comprehensive relation may be dependent on multiple factors $\left[W(i, m), n m_{i}, n k_{i}, \alpha_{i, m}\right.$, $\left.\beta_{i, k}, r_{i, m}, r_{i, k}\right]$, which is to be studied in and in detail further.

(3) There exists a cumulative effect for the innovation ability for academic influence of any paper and even the most important article. For example, it is seen from Figure 3 that the papers published during 19501957 for TD Lee have had the biggest contribution to innovation ability due to his work on the violation of parity law in weak interaction, so the highest peak can reach 9000 and 13500 for TD Lee and his team, respectively. That is why TD Lee could win the Nobel Prize for physics in 1957 and has had a long-term effect in all over the world. The other peaks for the latest papers are not of so high influence. 


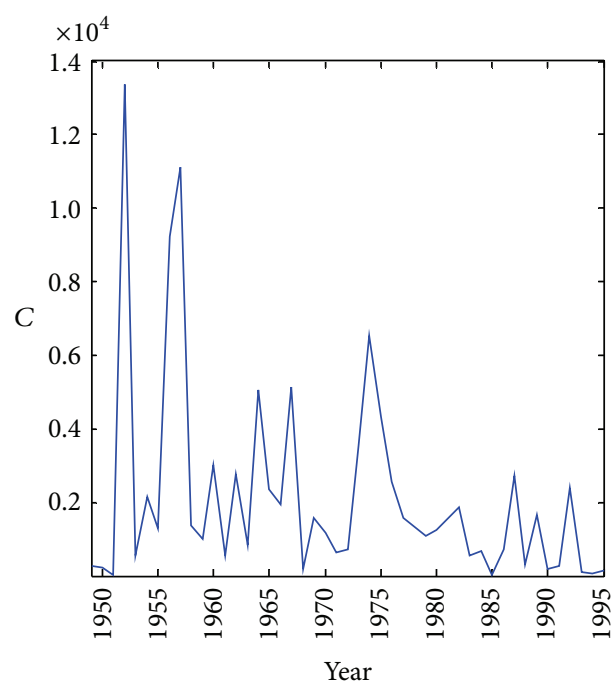

(a)

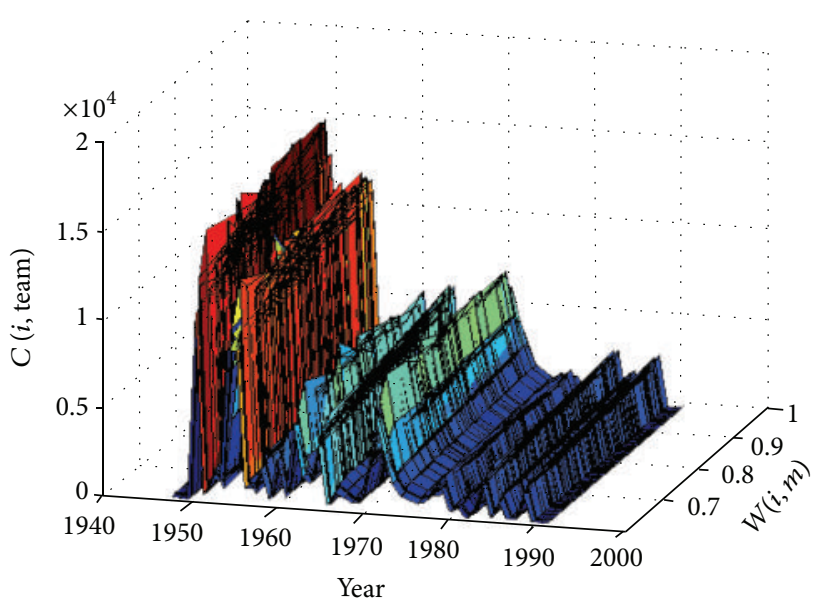

(b)

FIgURE 6: Innovation ability $C$ for TD Lee cooperation team. (a) $C$ versus year; (b) $(C(i$, team), $W(i, m))$ versus year.

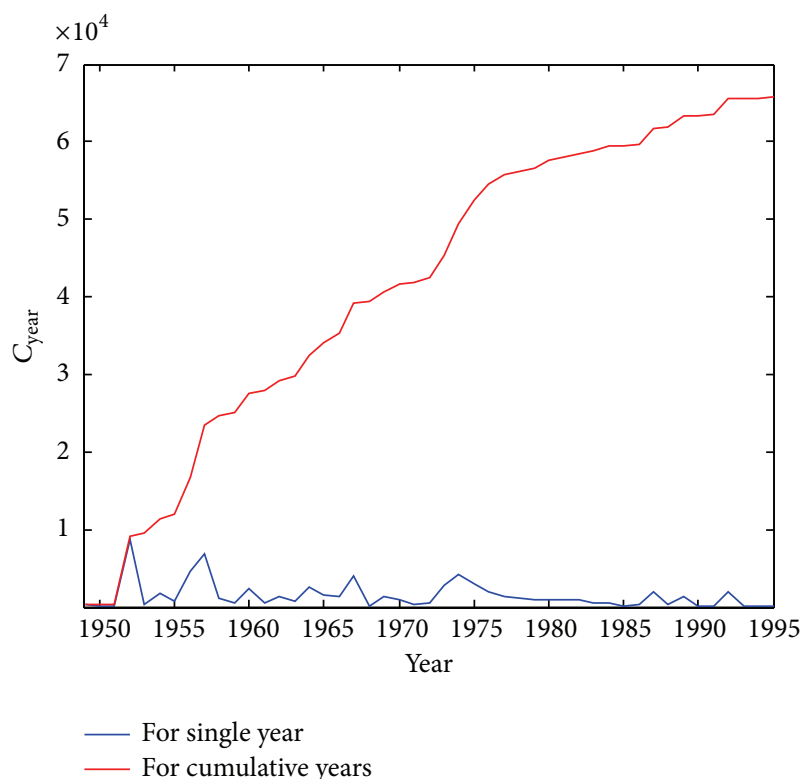

(a)

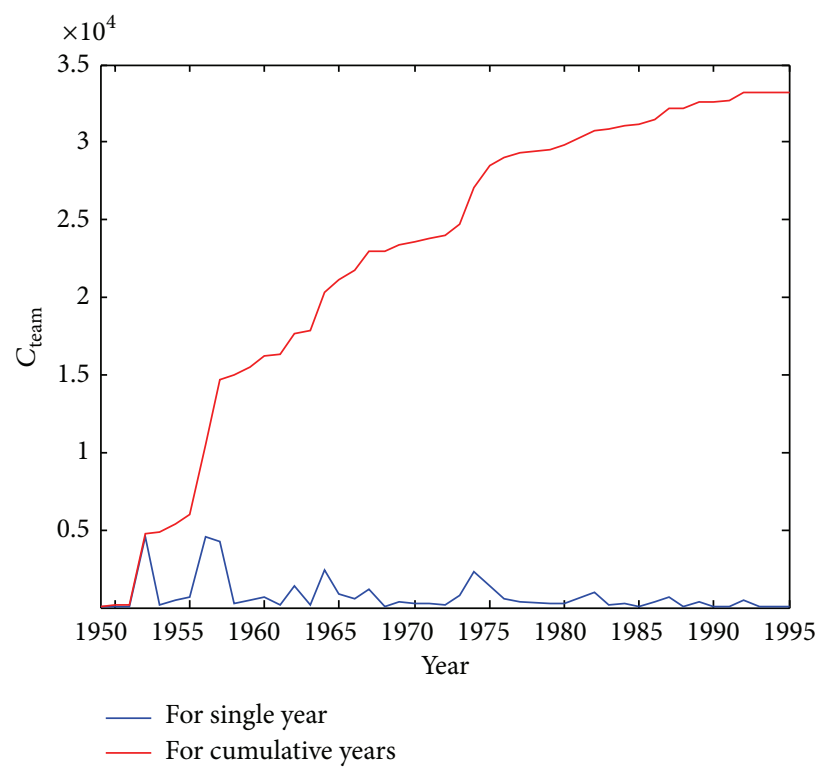

(b)

FIgURE 7: Comparison of the cumulative creative ability with single year creative ability. (a) For TD Lee; (b) For his team.

(4) The results above imply that the theoretical model can depict the creative ability for any nature scientist and his/her team, not being constrained by TD Lee and his team. Therefore, it has a certain universality, which may be extended to estimate the innovation ability for a nature scientist and his/her team.

From the above, the following conclusion can be drawn.

(1) It is found that TDLSCN not only has the common topological properties, both of scale-free and smallworld for a general scientist cooperation network, but also emerges the creation multiple-peak phenomenon for number of published paper with year evolution.

(2) The model of scientist innovation ability we proposed can provide a rational interpretation of new finding on creation multiple peak phenomenon, and this implies that there are some higher creation peaks in different working year for a lifetime of a nature scientist, and TD Lee scientist is a typical representative, so new finding has a certain universality for a nature scientist and maybe others.

(3) The model above may provide a better method for evaluating scientist innovation ability and offer a good 
promise for interdisciplinary analyses in the academic profession. The model is of application potential.

\section{Acknowledgment}

This work is supported by the Nature Science Foundation of China nos. 61174151 and 60874087.

\section{References}

[1] Q. Bi and J. Q. Fang, Network Science and Statistical Physics, Beijing University Press, Beijing, China, 2011.

[2] J. Q. Fang, X. F. Wang, Z. G. Zheng, Q. Bi, Z. R. Di, and X. Li, "New interdisciplinary science," Progress in Physics, vol. 27, no. 3, pp. 239-343, 2007 (Chinese).

[3] M. E. J. Newman, "Scientific collaboration networks. I. Network construction and fundamental results," Physical Review E, vol. 64, Article ID 016131, 8 pages, 2001.

[4] A. L. Barabási, H. Jeong, Z. Néda, E. Ravasz, A. Schubert, and T. Vicsek, "Evolution of the social network of scientific collaborations," Physica A, vol. 311, no. 3-4, pp. 590-614, 2002.

[5] A. Barrat, M. Barthélemy, R. Pastor-Satorras, and A. Vespignani, "The architecture of complex weighted networks," Proceedings of the National Academy of Sciences of the United States of America, vol. 101, no. 11, pp. 3747-3752, 2004.

[6] E. Almaas, B. Kovács, T. Vicsek, Z. N. Oltvai, and A. L. Barabasi, "Global organization of metabolic fluxes in the bacterium Escherichia coli," Nature, vol. 427, pp. 839-843, 2004.

[7] M. Li, D. Wang, Y. Fan, Z. Di, and J. Wu, "Modelling weighted networks using connection count," New Journal of Physics, vol. 8, p. 72, 2006.

[8] M. Li, J. Wu, D. Wang, T. Zhou, Z. Di, and Y. Fan, "Evolving model of weighted networks inspired by scientific collaboration networks," Physica A, vol. 375, no. 1, pp. 355-364, 2007.

[9] T. D. Lee, Digital Library: Selected Papers Vol. 1-4, http://localsev.lib.pku.edu.cn/lizhengdao/home/zhushu.asp.

[10] J. M. Soler, "A rational indicator of scientific creativity," Journal of Informetrics, vol. 1, no. 2, pp. 123-130, 2007.

[11] G. Wen, Z. Duan, G. Chen, and G. Xianmin, "A weighted localworld evolving network model with aging nodes," Physica A, vol. 390, no. 21-22, pp. 4012-4026, 2011. 


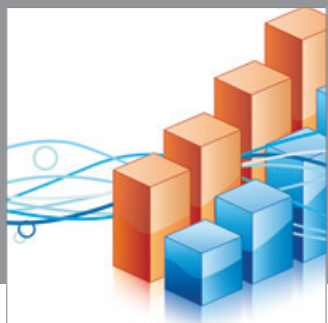

Advances in

Operations Research

mansans

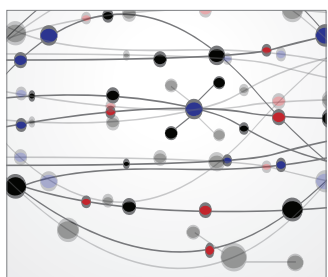

The Scientific World Journal
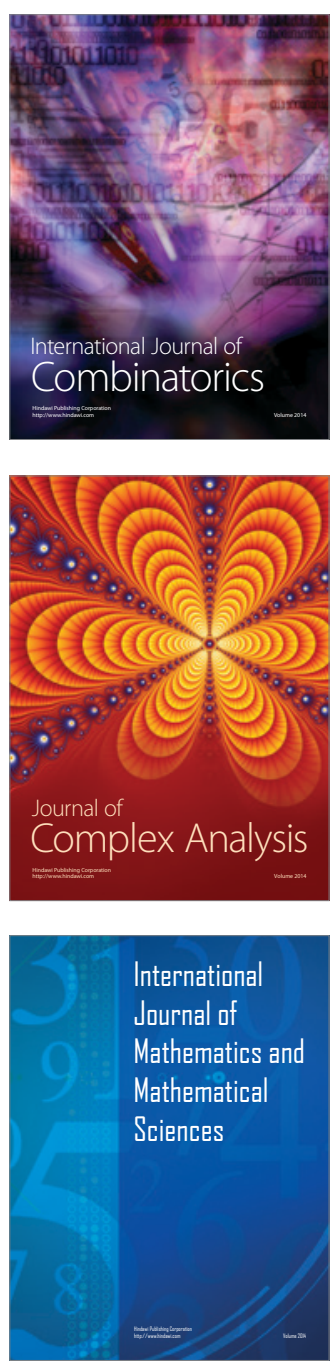
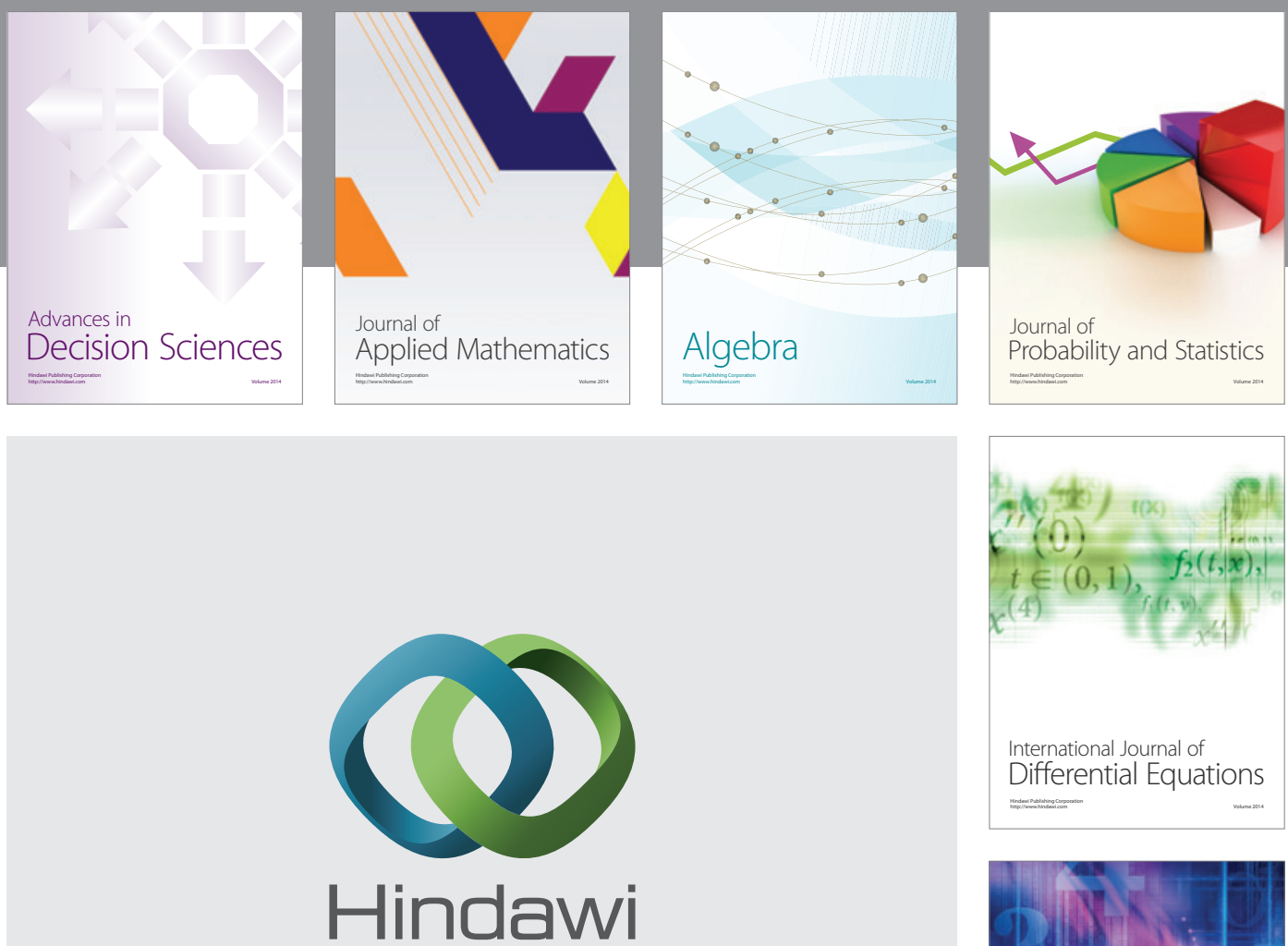

Submit your manuscripts at http://www.hindawi.com
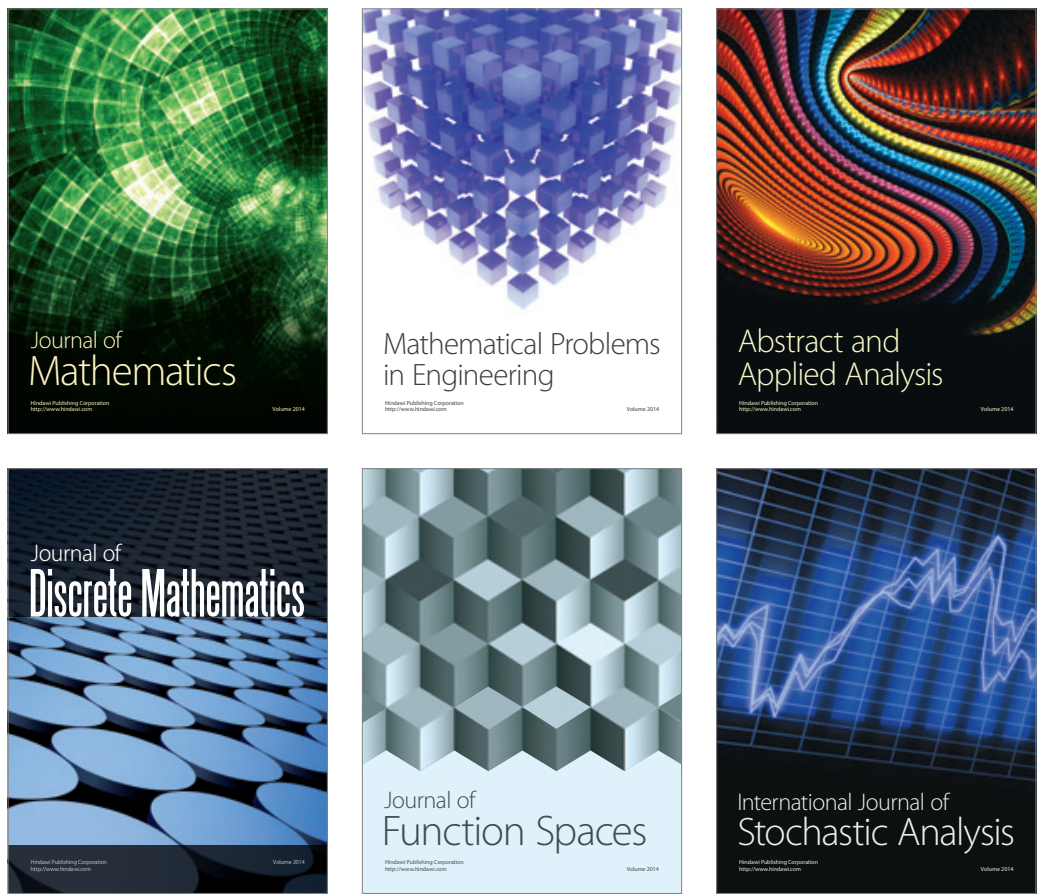

Journal of

Function Spaces

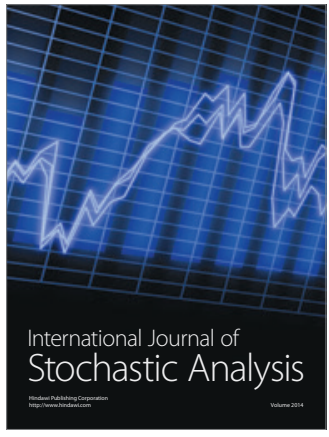

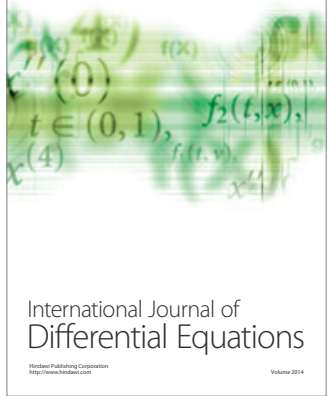
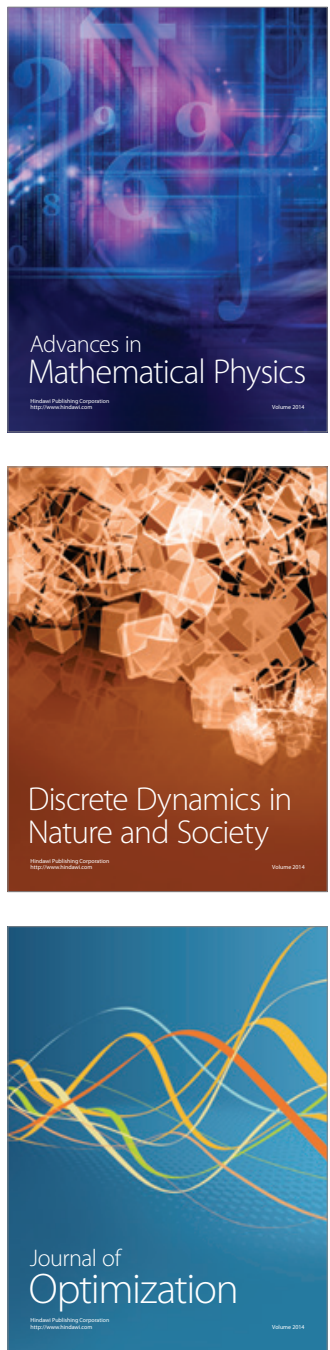